\title{
EDITORIAL
}

\section{Do anti-leukotriene agents inhibit asthmatic inflammation?}

\section{Introduction}

The 1982 Nobel Prize in Medicine or Physiology was awarded for the discovery that slow reacting substance, a contractile bioactivity long thought to be important in asthmatic bronchospasm, was comprised of a mixture of arachidonic acid 5-lipoxygenase (5-LO) metabolites, leukotrienes (LTs) $\mathrm{C}_{4}, \mathrm{D}_{4}$, and $\mathrm{E}_{4}$. Because all three of these lipids contain the amino acid cysteine, they are now collectively termed the 'cysteinyl LTs' (cysLTs). The recent development of drugs that inhibit the synthesis or actions of LTs ('anti-LT agents' or 'LT modifiers') represents a landmark bench-to-bedside triumph.

Although these drugs are currently used by millions of asthmatics world-wide, our knowledge of their pathophysiologic actions is still rapidly evolving. As 30 years worth of inhaled corticosteroid use has not been sufficient to address all conceivable questions about this class of agent, it cannot be surprising that we know far less about anti-LT agents. Of the many areas of uncertainty, one which has been regarded as pivotal, in view of the central importance of inflammation in asthma, is whether anti-LT agents are in fact anti-inflammatory. An article in this issue of Clinical and Experimental Allergy provides new insights into this question. The discussion that follows seeks to place these new findings into the evolving context of our understanding of the role played by LTs (and the effects of anti-LTs) in asthmatic inflammation.

\section{Leukotriene synthesis and receptors: the basics}

Biological effects of LTs require both the synthesis of these lipid mediators and the expression of receptors mediating their actions (see Fig. 1). LTs are synthesized primarily by inflammatory cells. Importantly, LT generation is amplified, via upregulation of enzymes in their biosynthetic pathway, by many other mediators implicated in the pathogenesis of asthma and allergic disease (reviewed in [1]); such mediators include IFN- $\gamma$, IL-4 and IL-5, granulocyte macrophage colony-stimulating factor (GM-CSF), eotaxin, endothelin, and TGF- $\beta$. It has recently been demonstrated that the adipocyte hormone leptin also increases LT biosynthesis [2]; since leptin is secreted in relation to body fat stores, this mechanism could contribute to the increased incidence of asthma that is associated with obesity. Two G protein-coupled receptors for cysLTs have been characterized, cysLT1 and cysLT2. CysLT1 is the best understood of these and is the target for the cysLT receptor antagonists (LTRAs) now in widespread clinical use, including montelukast, zafirlukast, and pranlukast. Another important concept to have recently emerged is that expression of cysLT1 can also be up-regulated by cytokines, including IL-4, IL-5, and IFN- $\gamma$ (reviewed in [3]). Thus, these observations help to explain the involvement of cysLTs in both Th1 (e.g. viral infections) and Th2 (e.g. allergen exposure) predominant states.

\section{Leukotrienes in inflammation, remodeling, and immune responses}

What about the actions of LTs relevant to asthmatic inflammation? A wealth of clinical evidence supports the conclusion that anti-LT agents abrogate the eosinophilic inflammation so characteristic of allergic asthma. The paper by $\mathrm{Wu}$ and coworkers in the March issue of Clinical and Experimental Allergy [4] extends our understanding of how this occurs. They examined the effects of a 3-day pre-treatment with once-daily intravenous montelukast on the inflammatory response to inhaled ovalbumin in antigen-sensitized BALB/c mice. Using a dose of montelukast that attenuated antigen-induced eosinophil numbers in lavage fluid by $>90 \%$, they observed significant reductions in lung levels of IL-4, IL-5, IL-13, and eotaxin, in serum IL-5 and IgE levels, and in lung vascular expression of vascular cell adhesion molecule-1 (VCAM-1). By integrating data from both in vivo animal studies such as this one as well as in vitro studies, we can now conclude that cysLTs modulate eosinophils by both direct and indirect mechanisms (Fig. 1).

First among the direct effects, the LTs promote differentiation of eosinophilic precursors in the bone marrow [5]. Second, they promote eosinophilic recruitment to the airways via effects on both chemotaxis [6] and expression of adhesion molecules on eosinophils [7] as well as vessels [8]. Third, they inhibit eosinophil apoptosis [9], thereby prolonging the survival of those cells that reach the airways. The indirect effects of cysLTs reflect their ability to up-regulate gene expression for and/or release of various cytokines/chemokines that themselves promote eosinophil production, recruitment, and survival; these include IL-4, IL-5, IL-13, eotaxin, and GM-CSF [1]. Furthermore, these effects of cysLTs appear to exhibit some specificity for Th2 cytokines, as LT blockade or deficiency either fails to inhibit Th1 cytokines such as IFN- $\gamma$, or actually increases them [10-12].

Repeated antigen challenge to the rodent airway over a period of at least 8 weeks results not only in inflammation, but in structural changes such as mucus gland hyperplasia, basement membrane thickening, increased smooth muscle cell mass, and subepithelial fibrosis. These changes resemble those identified in the airways of human asthmatics and are commonly termed 'remodeling.' A possible role for cysLTs in airway remodeling (Fig. 1) is suggested by animal studies demonstrating that chronic administration of cysLT antagonists abrogate all of these alterations [13,14]. The recent report that 5-LO null mice are significantly protected from pulmonary parenchymal 


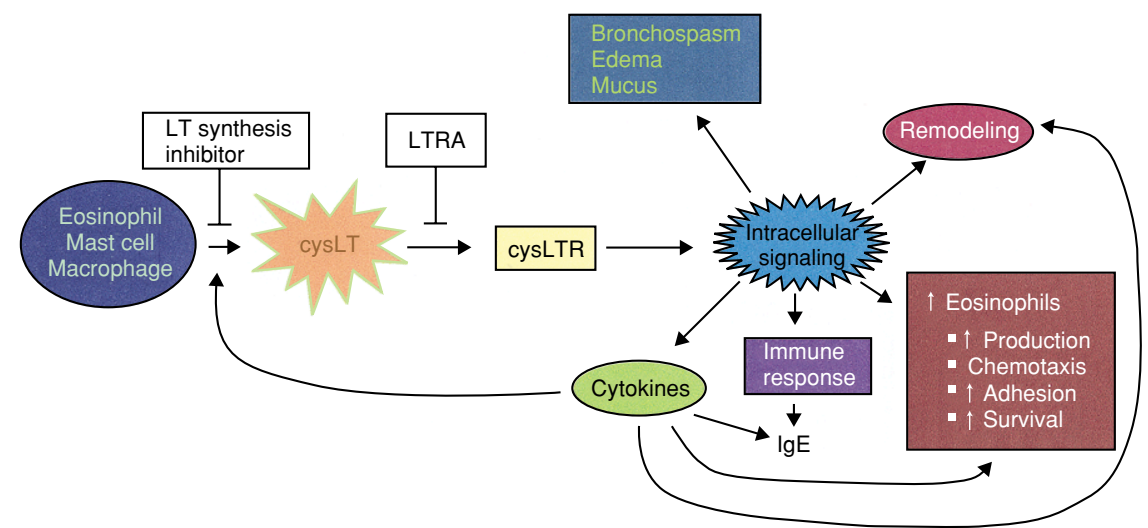

Fig. 1. Synthesis and actions of cysteinyl leukotrienes. CysLTs are synthesized by eosinophils, mast cells, and macrophages. Upon interaction with cysLT receptor(s), intracellular signals are generated in target tissues, which result in a variety of acute and chronic responses relevant to the pathophysiology of asthma. By inhibiting cysLT synthesis or antagonizing cysLT receptors (LTRA), anti-LT agents can modify these responses. fibrosis [10] adds further credence to the possibility that LTs are causal participants in tissue remodeling responses. Such a role could reflect their well-known effects on inflammatory cells as well as their less recognized effects on mesenchymal cells [15]. Whether anti-LT agents can abrogate airway remodeling in human asthmatics is not yet known, but is of crucial importance.

Moving from the airways to a more proximal step in the development of allergic inflammation, certain findings support a role for cysLTs in the initiation of immune responses (Fig. 1). Serum IgE levels reflect the magnitude of the systemic immune response to antigen, and it is intriguing that 5-LO null mice [16] as well as montelukast-treated mice [4] exhibit far less of an increase in serum IgE levels following antigen sensitization and challenge than control animals do. Dendritic cells are the professional cells responsible for antigen processing and presentation to lymphocytes, and they express 5-LO and its activating protein and synthesize LTs [17]. It has also been shown that dendritic cell trafficking to lymph nodes is, under some conditions, dependent on cysLT signalling [18]. Although the clinical applicability and significance of these findings have yet to be established, a potential role for LTs in initiating immune responses would have implications well beyond asthma.

\section{Intracellular events}

In addition to expanding the repertoire of LT effects, recent research has also delved into the intracellular mechanisms by which these mediators act (Fig. 1). A variety of intracellular signals have been shown to be activated in response to LTs. These include increases in intracellular calcium levels and reactive oxygen species, actin reorganization, and activation of kinases including protein kinase $\mathrm{C}$, mitogen-activated protein kinase, and phosphatidylinositol 3-kinase [19-21]. The ability of anti-LT drugs to suppress gene expression for cytokines and other mediators, discussed above, likely reflects their interruption of LT-induced signalling to the nucleus. A significant body of literature has implicated endogenous 5-LO metabolites as enhancers of the activation of such transcription factors as nuclear factor- $\kappa \mathrm{B}(\mathrm{NF}-\kappa \mathrm{B})$, NF-IL6, and AP-1 [22, 23], known to be crucial determinants of cytokine gene transcription. However, although compelling evidence links $\mathrm{LTB}_{4}$ to such activation, no evidence as yet implicates cysLTs themselves in this process.
In this issue of Clinical and Experimental Allergy, Tomari and colleagues [24], studying antigen-stimulated peripheral blood monocytes in vitro, present the first evidence that a cysLT1 LTRA, pranlukast, inhibits activation (i.e. nuclear translocation) of $\mathrm{NF}-\kappa \mathrm{B}$. This effect was associated with a parallel inhibition of TNF- $\alpha$ production. Moreover, pranlukast and low-dose dexamethasone exhibited additive inhibition of these processes. These results certainly extend the repertoire of LTRAs, but a few caveats must be raised. First, these effects of pranlukast are quite modest, and their biological significance is uncertain. At the same time, however, one can imagine that the amplifying effects of cysLTs on transcription factor activation might be more impressive in cell types with a greater capacity for cysLT synthesis, such as mast cells or eosinophils. The second important caveat relates to the conclusions drawn regarding the mechanism of action of pranlukast in this experimental model. Because exogenous $\mathrm{LTD}_{4}$ alone failed to lead to nuclear translocation of $\mathrm{NF}-\kappa \mathrm{B}$, the authors concluded that pranlukast may be acting through pathways distinct from cysLT1 antagonism. Of course, any drug has the potential to exert unexpected and non-mechanism-based actions, and the 5-LO inhibitor zileuton has been recently reported to unexpectedly act as a scavenger of peroxynitrite [25]. However, the suggestion of a cysLT1-independent mechanism is at this point premature. First, other LTRAs were not examined.

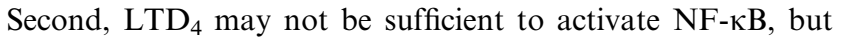
could still prime cells for enhanced antigen-induced activation. In a recent in vivo study, instillation of $\mathrm{LTD}_{4}$ alone into the airways of mice was without effect, but when co-administered with antigen, it amplified both antigen-induced eosinophil numbers and airway hyper-responsiveness [26]. It will surely be important to build upon these observations in order to clarify the role of cysLTs and of anti-LT agents in transcription factor activation in relevant experimental systems. Indeed, many other aspects of the intracellular receptors and mechanisms by which LTs act await further study [27].

\section{Controversies and conundrums regarding anti-leukotriene agents (and asthma)}

The incorporation of anti-LT agents into asthma treatment has not been without controversy. In particular, concerns about anti-inflammatory actions, therapeutic potency, and the 
interindividual heterogeneity of efficacy warrant some examination and challenge us to consider what this therapeutic class has taught us about asthma.

Do anti-LT agents inhibit asthmatic inflammation? Although I think the evidence is overwhelmingly positive, it must be acknowledged that there are pieces of data to the contrary. For example, these drugs have been noted in certain studies to not inhibit eosinophilic inflammation [28], IL-5 expression [26], and airway hyper-responsiveness [13], despite exerting other salutary effects in those same studies. These discrepancies may reflect the lack of correlation among various biomarkers of asthma, as discussed below. Moreover, it is hardly surprising that studies employing different types of subjects, species, cell types, or experimental conditions would yield discrepant results. Many negative findings can likewise be identified amidst the largely positive in the literature on inhaled corticosteroids. The real problem here is the term 'inflammation', which is no longer sufficiently precise to be very useful as we seek to better understand the pathophysiology of asthma. We will be better served by focusing on the effects of pharmacologic agents on specific biomarkers.

Finally, the study by Wu et al. [4] raises the intriguing possibility that current dosing of anti-LT agents may be inadequate for patients with severe chronic asthma or acute exacerbations. With the higher levels of both cysLTs and their receptors that are expected under such conditions, it would seem reasonable to re-examine dosing of a drug with as generous a safety margin as montelukast.

Since asthma is characterized by a complex network of cells and mediators, it is hardly surprising that intense scepticism surrounded the early development of a therapeutic approach targeting just one of these mediators. Why are these drugs, in fact, more effective than many would have predicted? Certainly, part of the answer lies in the capacity of LTs to modulate such a wide range of features central to the pathophysiology of asthma. These include actions that we have known about for decades - bronchospasm, airway oedema, and mucus secretion - as well as actions such as those discussed in this editorial. One of the most far-reaching principles to emerge from recent research is that an interplay exists between LTs and other mediators, such as cytokines. LTs both drive cytokine production and mediate certain cytokine actions. Such convergence of seemingly distinct biological circuits also provides a measure of optimism for other anti-mediator approaches to asthma therapy, including many that are currently in development.

While the basis for the heterogeneity of responsiveness to anti-LT therapy among individual patients requires further investigation, we can certainly speculate that it reflects the inevitable downside of a single mediator blockade approach in a multi-mediator disease. On the other hand, this phenomenon has also served to emphasize the heterogeneity of asthma itself. We now increasingly recognize that individual patients exhibit variable responsiveness even to inhaled corticosteroids [29] and inhaled $\beta$-adrenergics [30]. Furthermore, the clinical manifestations (e.g. symptoms, pulmonary function abnormality, bronchial hyper-responsiveness, and exacerbations) of asthma also vary among patients and the degree to which specific manifestations correlate with each other in any given patient is, at best, imperfect. This heterogeneity has profound implications for how we both treat and study the syndrome of asthma.

\section{References}

1 Peters-Golden M, Sampson A. Cysteinyl leukotriene interactions with other mediators and with glucocorticoids during airway inflammation. J Allergy Clin Immunol 2003; 111:S37-S48.

2 Mancuso P, Gottschalk A, Phare S, Peters-Golden M, Lukacs N, Huffnagle G. Leptin-deficient mice exhibit impaired host defense in gram-negative pneumonia. J Immunol 2002; 168:4018-24.

3 Holgate S, Peters-Golden M, Panettieri R, Henderson W. Roles of cysteinyl leukotrienes in airway inflammation, smooth muscle function, and remodeling. J Allergy Clin Immunol 2003; 111: S18-S36.

4 Wu A, Chik S, Chan A, Li Z, Tsang K, Li W. Anti-inflammatory effects of high-dose montelukast in an animal model of acute asthma. Clin Exp Allergy 2003; 33:359-66.

5 Braccione F, Dorman S, O'Byrne P et al. The effect of cysteinyl leukotrienes on growth of eosinophil progenitors from peripheral blood and bone marrow of atopic subjects. J Allergy Clin Immunol 2002; 110:96-101.

6 Spada C, Nieves A, Krauss A, Woodward D. Comparison of leukotriene $\mathrm{B}_{4}$ and $\mathrm{D}_{4}$ effects on human eosinophil and neutrophil motility in vitro. J Leukoc Biol 1994; 55:183-91.

7 Fregonese L, Silvestri M, Sabatini F, Rossi G. Cysteinyl leukotrienes induce human eosinophil locomotion and adhesion molecule expression via a cysLT1 receptor-mediated mechanism. Clin Exp Allergy 2002; 32:745-50.

8 Pedersen K, Bochner B, Undem B. Cysteinyl leukotrienes induce $\mathrm{P}$-selectin expression in human endothelial cells via a non-cysLT1 receptor-mediated mechanism. J Pharmacol Exp Ther 1997; 281:655-62.

9 Lee E, Robertson T, Smith J, Kilfeather S. Leukotriene receptor antagonists and synthesis inhibitors reverse survival in eosinophils of asthmatic individuals. Am J Respir Crit Care Med 2000; 161:1881-6.

10 Peters-Golden M, Bailie M, Marshall T et al. Protection from pulmonary fibrosis in leukotriene-deficient mice. Am J Respir Crit Care Med 2002; 165:229-35.

11 Tohda Y, Nakahara H, Kubo H, Haraguchi R, Fukuoka M, Nakajima S. Effects of ONO-1078 (pranlukast) on cytokine production in peripheral blood mononuclear cells of patients with bronchial asthma. Clin Exp Allergy 1999; 29:1532-6.

12 Nag S, Lamkhioued B, Renzi P. Interleukin-2-induced increased airways responsiveness and lung Th2 cytokine expression occur after antigen challenge through the leukotriene pathway. Am J Respir Crit Care Med 2002; 165:1540-5.

13 Henderson W Jr, Tang L-O, Chu S-J et al. A role for cysteinyl leukotrienes in airway remodeling in a mouse asthma model. Am J Respir Crit Care Med 2002; 165:108-16.

14 Wang CT, Xu L, Martin J. Role of leukotriene D4 in allergen-induced increases in airway smooth muscle in the rat. Am Rev Respir Dis 1993; 148:413-7.

15 Panettieri R, Tan E, Ciocca V, Luttmann M, Leonard T, Hay D. Effects of LTD4 on human airway smooth muscle cell proliferation, matrix expression, and contraction in vitro: differential sensitivity to cysteinyl leukotriene receptor antagonists. Am J Respir Cell Mol Biol 1998; 19:453-61.

16 Irvin C, Tu Y-P, Sheller J, Funk C. 5-Lipoxygenase products are necessary for ovalbumin-induced airway responsiveness in mice. Am J Physiol (Lung Cell Mol Physiol) 1997; 272: L1053-8.

17 Harizi H, Juzan M, Moreau J-F, Gualde N. Prostaglandins inhibit 5-lipoxygenase-activating protein expression and leukotriene $\mathrm{B}_{4}$ production from dendritic cells via an IL-10-dependent mechanism. J Immunol 2002; 169:139-46.

18 Robbiani D, Finch R, Jager D, Muller W, Sartorelli A, Randolph G. The leukotriene $\mathrm{C}_{4}$ transporter MRP1 regulates CCL19 (MIP-3 $\beta$, 
ELC)-dependent mobilization of dendritic cells to lymph nodes. Cell 2000; 103:757-68.

19 Hoshino M, Izumi T, Shimizu T. Leukotriene $\mathrm{D}_{4}$ activates mitogen-activated protein kinase through a protein kinase $\mathrm{C} \alpha$-Raf-1-dependent pathway in human monocytic leukemia THP-1 cells. J Biol Chem 1998; 273:4878-82.

20 Saegusa S, Tsubone H, Kuwahara M. Leukotriene $\mathrm{D}_{4}$-induced rho-mediated actin reorganization in human bronchial epithelial cells. Eur J Pharmacol 2001; 413:163-71.

21 Thodeti C, Nielsen C, Paruchuri S, Larsson C, Sjolander A. The epsilon isoform of protein kinase $\mathrm{C}$ is involved in regulation of the $\mathrm{LTD}_{4}$-induced calcium signal in human intestinal epithelial cells. Exp Cell Res 2001; 262:95-103.

22 Stankova J, Rola-Pleszczynski M. Leukotriene $\mathrm{B}_{4}$ stimulates $c$-fos and c-jun gene transcription and AP-1 binding activity in human monocytes. Biochem J 1992; 282:625-9.

23 Anthonsen M, Andersen S, Solhaug A, Johansen B. Atypical $\lambda / \mathrm{t}$ PKC conveys 5-lipoxygenase/leukotriene $\mathrm{B}_{4}$-mediated cross-talk between phospholipase $\mathrm{A}_{2} \mathrm{~s}$ regulating $\mathrm{NF}-\mathrm{\kappa} \mathrm{B}$ activation in response to tumor necrosis factor- $\alpha$ and interleukin-1 $\beta$. J Biol Chem 2001; 276:35344-51.

24 Tomari S, Matsuse H, Machida I et al. Pranlukast, a cysteinyl leukotriene receptor 1 antagonist, attenuates allergen specific $\mathrm{TNF}-\alpha$ production and $\mathrm{NF}-\kappa \mathrm{B}$ nuclear translocation in peripheral blood monocytes from atopic asthmatics. Clin Exp Allergy 2003; 33:795-801.

25 Coffey M, Phare S, Peters-Golden M. Peroxynitrite-induced nitrotyrosination of proteins is blocked by direct 5-lipoxygenase inhibitor zileuton. J Pharmacol Exp Ther 2001; 299:198-203.
26 Eum S-Y, Maghni K, Hamid Q, Campbell H, Eidelman D, Martin J. Involvement of the cysteinyl-leukotrienes in allergen-induced airway eosinophilia and hyperresponsiveness in the mouse. Am J Respir Cell Mol Biol 2003; 28:25-32.

27 Bandeira-Melo C, Woods L, Phoofolo M, Weller P. Intracrine cysteinyl leukotriene receptor-mediated signaling of eosinophil vesicular transport-mediated interleukin-4 secretion. J Exp Med 2002; 196:841-50.

28 Diamant Z, Grootendorst D, Vselic-Charvat $M$ et al. The effect of montelukast (MK-0476), a cysteinyl leukotriene receptor antagonist, on allergen-induced airway responses and sputum cell counts in asthma. Clin Exp Allergy 1999; 29:42-51.

29 Szefler S, Martin R, King T et al. Significant variability in response to inhaled corticosteroids for persistent asthma. J Allergy Clin Immunol 2002; 109:410-8.

30 Strauss L, Hejal R, Galan G, Dixon L, McFadden ER Jr. Observations on the effects of aerosolized albuterol in acute asthma. Am J Respir Crit Care Med 1997; 155:454-8.

M. Peters-Golden Division of Pulmonary and Critical Care Medicine University of Michigan Health System Ann Arbor, Michigan

USA

E-mail:petersm@umich.edu 